\title{
Evidence for a Causal Relationship Between Mach's Principle and the Quantitative Latency for Universal Entanglement
}

\author{
Michael A. Persinger*, Stanley A. Koren \\ Laurentian University, Sudbury, Ontario P3E 2C6, Canada \\ *E-mail address: mpersinger@laurentian.ca
}

\begin{abstract}
Support for Mach's principle of the Prominence or the Immanence of the Universe which states that the behavior of any part of the Cosmos is determined by all of its parts requires quantitative convergence from the appropriate combination of universal parameters. Applications of recent calculations based upon a diffusivity with a real value $\left(\sim 10^{23} \mathrm{~m} \cdot \mathrm{s}^{-1}\right)$ revealed solutions that are consistent with the concept of dragging inertial frames and geodetic (Lense-Thirring) precession. The latency for non-local entanglement around the earth is similar to frame dragging as measured by Ciufolini. The independent solution for this real value to capture Mach's "whole universe" requires the contribution from quantum-level Zero Point Fluctuations. Consideration of this value for solarterrestrial distances could explain the non-local "gravitational" effects upon biological reactions that appear to precede electromagnetic effects. The verification of the "entanglement latency" by independent methods could produce the validation for Mach's principle.
\end{abstract}

Keywords: Mach's principle; entanglement latency; $10^{23} \mathrm{~m} \cdot \mathrm{s}^{-1}$; inertial frame dragging; gravitationalelectromagnetic differentiation

\section{INTRODUCTION}

Mach's principle states that the behaviour of any part of the Cosmos is determined by all of its parts [1]. More specifically Mach assumed that forces of inertia and centrifugal changes were due to accelerations and rotations with respect to all masses of the universe. According to classic relativity theory, centrifugal and inertial forces are caused by derivative functions from rates of change, such as accelerations or rotations referenced to local conditions. These local inertial time frames are influenced by the dynamic changes in massenergy within the universe.

These frames of reference are assumed to be dragged by the motion and rotation of proximal matter. According to Einstein's general theory of relativity a spinning mass will drag inertial frames with this motion [2,3]. One consequence of this process is the distortion of the "flow of time" around a spinning body. Recently Ciufolini [3] reviewed the quantitative evidence for frame dragging according to satellites orbiting the Earth.

The associated precession through transport of the spin vector in curved space-time near a substantial mass is considered to be a "hyperfine precession" due to the interaction between 
the angular momenta of the two spins of the two objects [2]. Hence geodetic precession and Lense-Thirring drag are due to intrinsic angular momentum that shares a common source of variance which has been termed gravitomagnetism.

For two clocks moving in opposite directions around the earth at an attitude of 6,000 $\mathrm{km}$, the difference in time after one revolution would be $\sim 10^{-16} \mathrm{~s}$ [3]. This value is the same order of magnitude as predicted by other physical parameters of universal diffusivity that may reflect an actual real value for the passive latency to produce "excess correlation" or entanglement. Evidence is presented here that this approximate temporal latency might be support for Mach's principle.

\section{ENTANGLEMENT LATENCY}

If Mach's principle is valid then there should be quantitative evidence derived from known constants and parameters applicable at universal frameworks. Dotta and Persinger [4] found that two spaces within the centers of two coupled magnetic fields that rotated counterclockwise around fixed circumferences with changing angular velocities produced excess correlations for about $8 \mathrm{~min}$ between two simultaneous photoluminescent reactions.

More precisely the condition to facilitate both the non-zero rest mass of the photon as well as a third state of polarization in which the vector of the electric field orients along the line of motion [5] was produced by placing each of the two photon reactions in the centers of arrays of 8 solenoids that were activated in both loci simultaneously. The duration of the field in the first solenoid was $20 \mathrm{~ms}$ followed by an acceleration of $2 \mathrm{~ms}$ for each subsequent solenoid until the duration in the $8^{\text {th }}$ solenoid was $6 \mathrm{~ms}$. For the decelerating condition $2 \mathrm{~ms}$ was added to each solenoid such that the $8^{\text {th }}$ solenoid was activated for $34 \mathrm{~ms}$.

Because the circumference of the array was about $60 \mathrm{~cm}$, the average velocities for a single "rotation" were $5.8 \mathrm{~m} \cdot \mathrm{s}^{-1}$ and $2.8 \mathrm{~m} \cdot \mathrm{s}^{-1}$, respectively. The numbers of rotations were either $9.6 \mathrm{~Hz}$ or $4.6 \mathrm{~Hz}$, respectively. However the durations of the field in each of the 8 solenoids changed systematically. Consequently there was an implicit rate of rate of change (an additional acceleration).

Mach's concept indicated that centrifugal and inertial forces are due to rotations and accelerations with respect to all masses in the Universe. In the Dotta and Persinger [4] experiment the phase-velocity of electromagnetic energy was dissociated from the group velocity (movement around the circle). This process would allow theoretically the photon to display a non-zero mass [5]. The excess correlation only occurred if both reactions were first exposed for about 6 min to an accelerating group velocity of a decelerating phase-modulated field followed by exposure for $12 \mathrm{~min}$ to a decreasing group velocity with an accelerating phase-modulated field. All other combinations of group and phase velocities were not effective.

If the chemical reactions shared the same "spinning time frames" driven by acceleration and then deceleration of the rate of rotation, the photon emission's doubled for synchronized injections of reactant. Functionally the two loci behaved as if they were the same space such that the output was twice the output of any single locus. The apparent transposition of axes for three dimensional and potentially Minkowski space is a condition for the excess correlation that facilitates entanglement.

In the pursuit of accommodating experimental evidence from putative local examples of excess correlation or entanglement, Persinger and Koren [5] employed dimensional analyses to discern the equivalence for a metric of four-dimensional space, the product of $2 \pi r, 4 \pi r^{2}$, 
$4 / 3 \pi r^{3}$ and $2 \pi r f$, where is $f$ is frequency. The value is $21.3 \pi^{4} r^{7} f$. The dimensional aggregate is $\mathrm{m}^{7} \cdot \mathrm{s}^{-1}$.

The geometric constant $\left(2.13 \pi^{4}\right)$ has been shown to be a quantitative candidate to accommodate the discrepancy between measured and potential energy within sub-matter space [6]. We selected the formula for the most symmetrical closed boundary because motion within these infinite but bounded geometries would imply acceleration.

We [5] found one combination of basic parameters within the universe whose solution produced this aggregate:

$$
21.3 \pi^{4} r^{7} f=G^{2} \cdot m^{2} \cdot d \cdot t^{3}
$$

where $\mathrm{G}$ is the Newtonian Gravitational Constant, $\mathrm{m}=$ the mass of the universe, $\mathrm{d}=$ diameter of visible universe, and $\mathrm{t}=$ the age of the universe in seconds. In this instance $\mathrm{m}=2.38 \cdot 10^{52}$ $\mathrm{kg}, \mathrm{d}=8.86 \cdot 10^{26} \mathrm{~m}$ and $\mathrm{t}=4.06 \cdot 10^{17} \mathrm{~s}$.

The resulting value was $1.49 \cdot 10^{164} \mathrm{~m}^{7} \cdot \mathrm{s}^{-1}$. The $7^{\text {th }}$ root results in a hypothetical quality we considered to be analogous to "diffusion" with a quantitative value of $2.84 \cdot 10^{23} \mathrm{~m} \cdot \mathrm{s}^{-1}$. Assuming the modern estimates of the diameter of the universe this means that "diffusion" could occur throughout the universal volume from the furthest point between 7 and $8 \mathrm{~min}$. We concluded that this value would be a type of latency for the completion of the "excess correlation" [4].

\section{LOCAL EVIDENCE}

The diffusion velocity of $2.84 \cdot 10^{23} \mathrm{~m} \cdot \mathrm{s}^{-1}$ when applied to the circumference of the earth, $4 \cdot 10^{7} \mathrm{~m}$ indicates that the conditions for excess correlation would occur within $\sim 10^{-16} \mathrm{~s}$. This is precisely the order of magnitude of precession of two spinning bodies. Such precession has been attributed to the dragging of inertial frames of a spinning mass. The difference between two aging systems moving around the earth in opposite directions due to the Earth's spin would be approximately $10^{-16} \mathrm{~s}[2]$.

The congruence suggests that inertial frames can be "dragged" around a spinning body and influence the flow of time. This flow of time would therefore be highly correlated with or be an identity from the "diffusivity" of the "connectiveness" that relates the mass, age and width of the universe with each other and whatever medium is represented by G.

For comparison the latency for entanglement, employing equation (1) for a return distance to the sun $\left(3 \cdot 10^{11} \mathrm{~m}\right)$ would be $\sim 1 \cdot 10^{-12} \mathrm{~s}$. This value is remarkably similar to the central range of the life time of the hydronium ion (the water molecule with the additional proton) within water [7]. Previous calculations indicated that the average duration of the hydronium ion is coupled precisely to the diffusivity of the dynamics of water. The ratio of the proton's magnetic moment and unit charge produces a term which when multiplied by the average life-time of the hydronium ion results in an area. The linear distance is the space between water molecules [8].

Under normal conditions, the time required for electromagnetic fields to traverse the solar-terrestrial distance is about $8 \mathrm{~min}$. This duration of entanglement would suggest that solar phenomena could occur at earth distances in a non-local manner particularly for the movement of protons through the matrices of water molecules. Due to the counterclockwise rotation of the earth the interference patterns from the non-local coupling would be expected to be more prominent during sunrise rather than sunset. 
A functional connection between entanglement latency and the process that is associated with dragging inertial frames could accommodate empirical observations that have eluded explanation. For over 20 years Takata [9] observed that the sedimentation rate of protein in blood changed suddenly about 6 to 8 minutes before the moment of astronomical sun rise. This apparent "instantaneous" effect, if it reflected the entanglement diffusivity function, would have required only a picosecond to have been initiated.

The Novosibirsk Group, as reported by Klocheck et al [10] showed that the apparent coupling between physical and biological sensors occurred when the covered inlet aperture of the telescope was directed to lead the "visible" Sun by $2^{\circ} 4.6^{\prime}$. They concluded that the impact from the "true" Sun displays a speed that far exceeds the velocity of light.

Klocheck et al [10] developed a device to discern "non-electromagnetic" cosmic radiation that exhibited gravitational wave features. It was composed of precision quartz mechanical resonators with a working frequency of $5 \mathrm{MHz}$. They were vacuum-treated and shielded from external electromagnetic fields. These researchers found that one optimal condition for detecting these "T signals", which appear to be related to phenomena within the upper solar corona, occurred with discrete measurements of $10 \mathrm{ps}$. This is within the range predicted by the "diffusivity" relation for earth-solar distances.

These same researchers also found that the fluctuations in luminosity of photobacteria displayed a variance that was strongly correlated (0.9) with dispersions of the "gravitational" signal. This might be interpreted as a manifestation of excess correlation or "entanglement" between changes in numbers of photons from the sun and those generated within aqueous environment within which the photochemical reactions of the photobacteria occurred. In the balance of probabilities, the effect was mediated through the protons participating in the transient $\mathrm{H}_{3} \mathrm{O}^{+}$ion.

Within the human brain, one expects the manifestation of this effect would occur at Casimir distances. Casimir effects facilitate the transformation of virtual particles into real particles [12] assuming there is a changing boundary condition of the electromagnetic field. The dynamic volume of the human brain can be considered a complex of changing boundary conditions that range from the entwined rostral-caudal waves moving coherently at $\sim 4.5 \mathrm{~m} \cdot \mathrm{s}^{-1}$ over the cerebral cortices [13] to the rotating electromagnetic field that moves along the axon barrel of each neuron towards the synapse.

Assuming there are about $10^{12}$ to $10^{14}$ synapses within the human cerebral cortices and each synapse is $1 \mu \mathrm{m}^{2}$ the total surface area of synapses within a topological field would be between $10^{0}$ to $10^{2} \mathrm{~m}^{2}$ or a linear distance between $1 \mathrm{~m}$ and $10 \mathrm{~m}$. These values divided by the latency to "entanglement" results in temporal intervals between 0.35 and $3.5 \cdot 10^{-23} \mathrm{~s}$. The equivalent energy within the quantum range can be obtained by dividing these values into Planck's constant $\left(6.626 \cdot 10^{-34} \mathrm{~J} \cdot \mathrm{s}\right)$. The resulting energy is between $\sim 19$ and $6 \cdot 10^{-11} \mathrm{~J}$ or a mass equivalent between 0.2 and $\sim 7 \cdot 10^{-27} \mathrm{~kg}$. This value is sufficiently close to the mass of a single proton $\left(1.67 \cdot 10^{-27} \mathrm{~kg}\right)$, such as the ones that contribute to $\mathrm{H}_{3} \mathrm{O}^{+}$, to pursue further.

Considering the numbers of protons within a human cerebral volume the manifestation of a single proton from Casimir forces might be considered insignificant. If one proton leaves a hydronium ion after $\sim 10^{-12} \mathrm{~s}$ and the water content of the cerebral cortices is about $300 \mathrm{cc}$ (18 cc per Mole) with a $\mathrm{pH}$ of about 7.4, the numbers of protons would be $\mathrm{M}=10^{-\mathrm{pH}}$ or $4 \cdot 10^{-8}$ $\mathrm{M}$ which is $\sim 4 \cdot 10^{17}$ molecules within that volume.

However, information associated with that transformation from a virtual to a real particle could influence the water molecules within meaningful biological time. Assuming no redundant inclusions in a hydronium ion for that proton, contact with most of the water molecules would occur within about 5 days $\left(\left(\sim 10^{12}\right.\right.$ shifts $\left.\left.\cdot \mathrm{s}^{-1}\right) \cdot\left(8.64 \cdot 10^{4} \mathrm{~s} \cdot \mathrm{d}^{-1}\right)\right)$. The sequential 
production of a virtual-to-real proton every second would decrease this time span. Thus this single "entanglement" would have the potential to affect some subset of the aqueous environment within which all of the chemical reactions that determine brain function occur.

\section{SHARED INERTIAS}

The essence of Mach's principle involves inertia or momentum $\left(\mathrm{kg} \cdot \mathrm{m} \cdot \mathrm{s}^{-1}\right)$. For this to be distributed throughout space in order for the inertias of any mass to be affected by all masses, there should be a pervasive medium reflected by the Gravitational Constant $\mathrm{G}\left(\mathrm{m}^{3} \cdot \mathrm{kg}^{-1} \mathrm{~s}^{-2}\right)$. This results in an aggregate composed of $\mathrm{m}^{4} \cdot \mathrm{s}^{-3}$. The multiplication by a fundamental frequency $\left(\mathrm{s}^{-1}\right)$ would permit a value after the $4^{\text {th }}$ root that is $\mathrm{m} \cdot \mathrm{s}^{-1}$.

Assuming a mass of the universe of $2.38 \cdot 10^{52} \mathrm{~kg}$ [14], a radius of $4.43 \cdot 10^{26} \mathrm{~m}$, and an age of $4.06 \cdot 10^{17} \mathrm{~s}$, the value $\mathrm{kg} \cdot \mathrm{m} \cdot \mathrm{s}^{-1}$, momentum, for the system would be $2.6 \cdot 10^{61} \mathrm{~kg} \cdot \mathrm{m} \cdot \mathrm{s}^{-1}$. When multiplied by the condition in which the momentum occurs, $\mathrm{G}\left(6.67 \cdot 10^{-11} \mathrm{~m}^{3} \cdot \mathrm{kg}^{-1} \mathrm{~s}^{-2}\right)$, the resulting value is $17.32 \cdot 10^{50} \mathrm{~m}^{4} \cdot \mathrm{s}^{-3}$. The significance of this value becomes apparent when the cut-off frequency of the Zero Point Fluctuation [15], 3.23 $10^{43} \mathrm{~s}^{-1}$ is multiplied to produce $56 \cdot 10^{93} \mathrm{~m}^{4} \cdot \mathrm{s}^{-4}$. The $4^{\text {th }}$ root of this value is $4.9 \cdot 10^{23} \mathrm{~m} \cdot \mathrm{s}^{-1}$.

This value is within empirical error measurement of the value obtained by setting the complex geometry of four-dimensional space equal to optimal combinations of universal parameters and indicators. From the perspective of Mach's principle, the derivation of this value indicates that one means by which any mass is affected by all masses of the universe might require their shared exposure to $G$ and to the intrinsic vibration of the level of space from which Zero Point Fluctuations and Potentials emerge [15].

One of the more probationary concepts entertained from time to time over the last century of atomic physics is the consequences of the sudden reversal of the direction (rather than the inversion of spin) of the electron within its circular orbit. At a more macroscopic level the "sudden reversal" in the direction of bulk electrons within a specifically structured linear conductor is fundamental to antenna-based transmission of electromagnetic fields.

The time required for one orbit of a Bohr electron moving at the fine structure velocity of $2.19 \cdot 10^{6} \mathrm{~m} \cdot \mathrm{s}^{-1}$ is about $1.52 \cdot 10^{-16} \mathrm{~s}$. This is the same duration required for a photon moving just below the velocity of light in a vacuum to traverse the $10 \mathrm{~nm}$ plasma cell membrane of the neuron. If the velocity shifted direction within one orbital time the result in one second would be $\left.\left[1 \mathrm{~s} \cdot 2\left(2.19 \cdot 10^{6} \mathrm{~m} \cdot \mathrm{s}^{-1}\right)\right] \cdot 1.5 \cdot 10^{-16} \mathrm{~s}\right)$ or $2.9 \cdot 10^{22} \mathrm{~m} \cdot \mathrm{s}^{-1}$. Although this is a factor of 10 slower than the diffusivity value, if the change occurred within $\tau \cdot(4 \pi)^{-1}$, where $\tau=$ the duration of an orbit, the value for the solution would be very similar.

\section{CONVERGENT EXPERIMENTAL SOLUTIONS}

Angular momentum is traditionally defined as the product of the moment of inertia and the angular velocity. In the experimental procedure [4] where the group velocity of magnetic flux volumes moved counterclockwise and in saltatory increments around a circular array of eight equally spaced solenoids, we assumed an angular momentum was produced.

The electronics of the solenoids (SPST-5VDC Reed Relays from Radio Shack) operated at $2.5 \mathrm{~V}$ with a coil resistance of $250 \Omega$, or $10 \cdot 10^{-3} \mathrm{~A}$. The approximate numbers of electrons, obtained by dividing the latter value by $1.6 \cdot 10^{-19} \mathrm{~A} \cdot \mathrm{s} \cdot \mathrm{q}^{-1}$ where $\mathrm{q}$ is electron, is $6.25 \cdot 10^{16}$. The classical mass of the electron is $9.1 \cdot 10^{-31} \mathrm{~kg}$; therefore the "total mass" would be $5.7 \cdot 10^{-14}$ 
$\mathrm{kg}$. The moment of inertia (assuming a radius of $9.6 \cdot 10^{-2} \mathrm{~m}$ for a circumference of $60 \mathrm{~cm}$ ) would be $5.25 \cdot 10^{-16} \mathrm{~kg} \cdot \mathrm{m}^{2}$ while the angular velocity $\left(\mathrm{v} \cdot \mathrm{r}^{-1}\right.$ ) for $2.8 \mathrm{~m} \cdot \mathrm{s}^{-1}$ (see section 2) would be: $0.29 \cdot 10^{2} \mathrm{~s}^{-1}$. The resulting angular momentum is $1.5 \cdot 10^{-14} \mathrm{~kg} \cdot \mathrm{m}^{2} \cdot \mathrm{s}^{-1}$.

Multiplication of the intrinsic frequency of this rotation, $4.6 \mathrm{~Hz}$, results in a unit of energy: $7 \cdot 10^{-14} \mathrm{~J}$. If the square of the approximately 2.7 to $2.8 \cdot 10^{8} \mathrm{~m} \cdot \mathrm{s}^{-1}$ expected for movement of electron energy in conductors is assumed rather than "c", the mass equivalent for that increment of energy would be $\sim 9 \cdot 10^{-31} \mathrm{~kg}$. This value is within measurement error of the classical mass of the electron and its Compton wavelength. Consequently our experimental procedure that produced the evidence of "excess correlations" that lead to the solutions to support Mach's contention might be extended to pursue the ramifications of local applications.

\section{CONCLUSIONS}

The convergence of a diffusivity value that might define the latency for non-locality or excess correlation anywhere in the universe with application of universal parameters to momentum and inertia strongly supports Mach's Principle. The possibility there is a distinct value $\left(\sim 10^{23} \mathrm{~m} \cdot \mathrm{s}^{-1}\right)$ for the latency required for excess correlation to occur which would allow any part of the universe to be influenced by another within about 6 to 8 minutes could validate Mach's contention. However it requires contributions from the smallest level of discourse where virtual matter and gravitational phenomena originate.

\section{References}

[1] J. Singh, Great Ideas and Theories of Modern Cosmology Dover, N.Y., 1961.

[2] N. Ashby, B. Shahid-Saless, Physical Review D 42 (1990) 1118-1122.

[3] I. Ciufolini, Nature 449 (2007) 41-47.

[4] B. T. Dotta, M. A. Persinger, Journal of Biophysical Chemistry 3 (2012) 72-80.

[5] L-C. Tu, J. Luo, G. T. Gillies, Reports on Progress in Physics 68 (2005) 77-130.

[6] M. A. Persinger, S. A. Koren, The Open Astronomy Journal 6(2013) 10-13.

[7] M. A. Persinger, International Letters for Chemistry, Physics and Astronomy 11 (2014) $18-23$.

[8] T. E. DeCoursey, Physiological Reviews 83 (2002) 476-579.

[9] M. A. Persinger, International Letters of Chemistry, Physics and Astronomy 2 (2014) $1-10$.

[10] M. Takata, Archives fur Meterologie, Geophysics and Bioklimatologie Series B 2 (1951) 20-30.

[11] N. V. Klochek, L. E. Palamarchuk, M. V. Nikonova, Biophysics 40 (1995) 883-891.

[12] M. Bordag, U. Mohideen, V. M. Mostepanenko, Physics Reports 353 (2001) 1-205. 
[13] R. Llinas, U. Ribary, Proceedings of the National Academy of Sciences 90 (1993) 2078-2081.

[14] M.A. Persinger, Journal of Physics, Astrophysics and Physical Cosmology 3 (2009) 1-3.

[15] H. E. Puthoff, Physical Review A 39 (1989) 2333-2342.

( Received 17 May 2014; accepted 29 May 2014) 